know of no way of distinguishing this before puncture, but the nature of the fluid will determine it; for if the fluid have been secreted from the tunica vaginalis, it will be found highly albuminous, coagulating by heat and nitric acid, while the fluid of the more superficial cysts contains a very slight trace of albumen. There is another point you must ascertain before operating, and that is, whether the hydrocele be complicated with hernia. Your patient states that he had the usual well-known symptom of hernia, and you find the posterior portion of the tumor opaque. Well, even if the hernia be irreducible, there is no danger in the palliative treatment, if you take due caution; but it is better not to inject, for fear of inflammation extending to the hernial sac. You would also be satisfied with the palliative means in very old people, whose constitutions it is found are incapable of supporting acute local inflammation; and you will sometimes be called to operate on persons of a very advanced age. J remember an old Norfolk admiral coming to me with a very large hydrocele, and he was either 89 or 90 years of age ; so, before puncturing, I asked him if it gave him much pain. "Oh! no," said he, "but Mrs. H. finds it very inconvenient" (loud laughter). Neither should I inject a hydrocele, the first time of tapping; for it often happens, though the patient may have complained of very little pain, that the mere irritation of the canula sets up an altered action sufficient to effect a permanent cure. Let me again impress upon you, in all cases, to attend to the position of the testicle, and not conclude, because you tapped with perfect safety six months ago, you can do so again. Just suppose a man comes into your room, and says, in an off-hand manner, he wants to be tapped again; don't simply seek for the cicatrix of your former puncture, and push in the trocar; for there may have been sufficient inflammation set up by your former operation to have caused adhesion of the testis to the front of the sac, and then, unless you take precaution, away goes the trocar straight into the substance of the testicle.

[The remainder of this lecture, on the radical cure of hydrocele, illustrated by several interesting cases, in our next number.]

\section{CONTRIBUTIONS TO THE PATHOLOGY OF CHILDREN.}

BY P. HENNIS GREEN, M.B.

Lecturer on Diseases of Children, at the Hunterian School of Medicine. (Continued from page 276.)

SCROFULOUS TUBERCLE OF THE BRAIN. CASE IX.

Sudden attack of epilepsy; paralysis, with trembling of the limbs; measles; death; tubercle of the cerebrum; softening of the spinal marrow.

- Landormi, 5 years of age, was admitted into hospital on the $2 \mathrm{~d}$ of September, 1833. He was immediately vaccinated, and the pock passed through its several stages in a regular manner. The child generally enjoyed good health. At the age of four he had the hooping-cough, which lasted six weeks only. Four months back lie was suddenly seized with an epileptic attack, followed by weakness of the left leg, which compelled him to use a crutch; the left arm also was weak, and almost paralysed, the patient being unable to raise it to his mouth. Three weeks afterwards the limbs on the right side of the body were paralysed in a similar manner; and at length the child was unable to use either his arms or his legs. Since the epileptic attack he has had constant accesses of fever, with vomiting at intervals, and trembling of the body, but chiefly of the limbs; he also frequently complained of pains in the head and loins. No cough or diarrhœa; appetite pretty good. The power of moving the arms had been recovered a few days before admission into hospital, and on the $2 \mathrm{~d}$ he had a fresh attack of epilepsy. Leeches had been applied to the anus; two blisters to the legs; the spine was rubbed with stimulating liniments.

Sept. 5 . The face is rather flushed, but the expression of the countenance is natural. Intellectual faculties quite clear. The sensibility and mobility of the upper extremities are not altered, but the lower extremities are extremely stiff, as also is the trunk. Pulse 100; skin not warm; the cutaneous sensibility appears to be increased; severe pain along the spine in the lumbar region, and also in the head; excretion of urine involuntary; bowels habitually constipated. Six cupping glasses along the spine; calomel, six grains, in three doses.

For the next few days the child continued in the same state. Occasionally the calomel produced a hard evacuation; the skin remained cool; the pulse varied from 95 to 100 .

On the 10th the child commenced coughing. On the 13th he complained much of his back; the spine was again examined, but no deviation or curvature was discovered; when placed on the abdomen, he was unable to turn on the back. Two setons were applied near the most painful part of the lumbar region.

16. Febrile symptoms manifested themselves to-day; the child coughed a good deal; on examining the chest some crepitating râle was heard on both sides behind the scapulæ; pulse 112. Six ounces of blood were now drawn from the arm. On the following day the pulse was 128 ; respiration 44 ; the blood was not covered with an inflammatory crust.

On the 18th the symptoms continued without any remarkable change; cough frequent, dry ; pulse 124 ; abdomen free from pain; no diarrhœa. The child was remarked to have sneezed frequently since yesterday.

19. An eruption of measles has appeared on the face and limbs. Eyes injected; cough dry and harsh; pulse 130.

20. The child has been delirious during the night. The whole body is very stiff; the lower extremities also rigid, particularly the right one; the right foot is strongly bent inwards. He has had romiting and diarrhœa, and vomited up two escarides; pulse 136 ; respiration 68 . Complains much of pain in the lumbar region. There are several papulæ on the back and loins.

21. Delirium continues; the eruption of measles is pale on the face; on the genital organs and thighs there are several pustules of varioloid; pulse 142 ; respiration 68 ; cough frequent, and in fits; souffle tubaire on the right side of the chest; tongue pale, and dry. Six leeches to the right side of the cliest; sinapisms to the feet.

22. The child is dying; convulsive movements of the limbs; face purplish. Death took place at 10 o'clock, A.M.

\section{Body examined 24 hours after Death.}

Head: The arachnoid lining the dura mater adheres to the cerebral serous membrane over the glands of Pacchioni, which are very large, and resemble small pearls in size. The arachnoid covering the middle third of the hemispheres, and in the great fissure, is opaque ; underneath the former is a flattened tubercular mass, about a quarter of an inch in thickness, and tuberculated on the upper surface. The tubercular matter extends over a great portion of the surface of the brain. The cerebral tissue underneath is softened to the depth of an inch, and contains several small clots of blood, not bigger than a lentil. There are several miliary tubercles in the pia mater; no effusion into the ventricles. Cerebellum healthy. At the level of the seventh and eighth dorsal vertebræ the spinal marrow is softened to the extent of an inch in length; the pia mater in several points contains miliary tubercles.

Chest: Right lung adherent. throughout to the chest; the upper and middle lobes are in a state of grey hepa-
tisation, and infiltrated with tubercular matter. There are 
some nodules of lobular pneumonia in the left lung, with a few tubercles. Heart healthy.

Ablomen: Stomach healthy. There are a few points of injection in the small intestines, but the mucous membrane is healthy; the digestive canal contains about twenty ascarides, and some cricocephali. There is a considerable number of the latter in the heart also. No tubercles in the abdominal cavity.

\section{CAse X.}

Gradual paralysis; headache; amaurosis ; convulsions ; small-pox; death; tubercle of the cerebellum; effusion into the ventricles.

Therese Verité, 4 years of age, was admitted into hospital on the 9th of November, 1836. Her mother is healthy, but her father died of consumption at the age of 36 ; her maternal uncle also died of phthisis at the age of 18. The child's head always appeared to be a little large; she spoke well at two years of age, and her intellectual faculties were then highly developed. About this period she caught the hooping-cough, which lasted a long time; did not complain much until ten months back, when the right arm was observed to lose its power, and soon afterwards she was unable to walk about as before; four months afterwards she had violent headaches with sonnolence, and for the last five months, according to the mother's account, she has completely lost her vision. In the beginning of November she was seized with convulsions; with stiffness of the limbs, particularly on the right side of the body; the accesses lasted about ten to fifteen minutes, and were repeated three or four times a day. Five months back she romited repeatedly, with headache; and the same symptoms have returned within the last few days, the headache always preceding the vomiting. Has passed blood per anum several times, and is subject to diarrhœa; the stouls have often been passed involuntarily for the last three months, but latterly she has been much constipated.

On examination, the head was found to be much developed, being more than twenty inches in circumference; from ear to ear twelve inches, with a considerable prominence of the parietal bones. The cervical ganglia are much enlarged, and form a hard mass under the left angles of the jaws; she answers correctly to all questions; complains of headache; hears well, but is completely blind; the eyeballs are constantly turned upwards; the pupils equally dilated and immovable. The movements of the upper extremities are somewhat impeded, but the child can use both hands well. The lower extremities are completely paralysed and contracted. The sensibility of both arms and legs is much diminished. Skin moderately warm; pulse 100 ; respiration 18; urine and fæces passed involuntarily.

This state continued without any change until the 26th of November, when the patient caught a confluent smallpox, and died in a few days.

On examination of the body after death, a tubercle, the size of a hen's egg, was found in the left lobe of the cerebellum, and in the same lobe were two others not larger than nuts; these adhered to the dura mater; the nervous substance surrounding the tubercles was somewhat softened. The lateral ventricles contained about six ounces of clear fluid. Tubercles in the lungs and abdomen; several ulcerations in the small intestine.

\section{CASE XI.}

Headache; convulsions; worms ; peculiar involuntary movements of the eyes; measles; death; tubercles of the cerebrum and cerebellum; effusion into the ventricles.

Maria Pisochet, 6 years of age, was admitted into hospital on the 4th of June, 1836. She has not been vaccinated. Her father and mother are healthy. She remained with her nurse until three nonths back; since then she has had aimost constant pain in head, and within the last eight days has had two attacks of convulsions, and repeated vomiting, with irregular movements of the eyes, and, finally, strabismus. She vomited some worms, and also passed some per anum, after having taken some vermifuge potion.

5. The patient lies on her back. The head is large, but can be supported with ease in any position; fontanelles are completely ossified. The child answers clearly all questions which are asked, and says that she feels great pain in the frontal region; the countenance is natural, but the eyeballs present a remarkable motion, as if conrulsive, from left to right; pupils moderately and equally dilated; sight clear; she distinguishes the different objects which we present to her. There is no pain or prominence along the spine; skin moderately warm; pulse small, regular, 92 ; respirations 24 ; cough slight; on percussion we find a clear sound over the whole chest; respiratory murmur is normal, but strong ; tongue clear and moist; no impediment to deglutition; no diviation of tongue, or convulsive motion of lips, or muscles of face; no change of sensibility in limbs; but the power of lower extremities seems to be diminished, as she cannot walk without assistance. (On the bed-ticket was written chronic hydrocephalus; it was changed to ocular chorea, and worms.) Half an ounce of Corsican moss in four ounces of water ; calomel, six grains, in three doses; purgative lavement, with two ounces of manna; and four leeches behind ears. During the day she passed five or six stools, most of them involuntary, but there were no worms in them.

6. Child appears less depressed this morning; she asked for an utensil to make water in ; says that she feels no pain in the head; answers clearly all questions asked; no vomiting; skin is cool; respiration tranquil, 20 ; pulse regular, 80 to 84 ; sight is clear, but the convulsive movements of the eyeballs continue. Continue calomel; half an ounce of moss in four ounces of water; hydromel.

7. No change worthy of notice. The calomel and moss are continued.

8. The child's intellect is perfectly clear; she answers distinctly to all questions asked, and presents nothing abnormal except the irregular movements of the eyes; skin cool ; pulse 72 ; she asks to eat, and desires to get up, but is unable to support herself on her legs; two stools since yesterday, but no worms ; she often demands the closestool without passing anything. Calomel, four grains.

10. She passed a worm.

11. She walks about in the wards; intellectual faculties perfect; no lesion of motility or sensibility whatever. Omit the Corsican moss.

13. Passed another worm; the convulsive movements of the eyes are less, and she walks better; no fever.

16. Nothing now remains except the irregular movements of the eyes, and an incomplete paralysis, which prevents her from walking without assistance; skin cool; pulse 80.

On the 22d she was seized with measles. On the 24th a very pale eruption appeared, and the breathing was extremely difficult, accompanied by great anxiety; respiration $6+$, broken by a short dry cough; pulse very quick, and skin hot; the ocular movements and paralysis continue the same; she complains of some pain in the head. She died on the 25th, at half past eight A.M.

\section{Body examined 24 hours after Death.}

Head: Cranium well formed; the dura mater and arachnoid healihy, pia mater injected at the posterior part, with a few granulations on the surface and at the base of the brain; near the longitudinal fissure, at the posterior third of the right hemisphere, there is a flat tubercle, nearly as large as a fourpenny piece, and on the left side three others, of the same size, between the convolutions; the other parts of the brain healthy; the lateral rentricles contain three ounces of clear serum; but the nervous substance is here intact. In the left lobe of the cerebellum there is a tubercular mass, divided by a deep 
fissure into two portions, each as large as a walnut; it is irregular on the surface, and of a greeris'? yellow colour; the surrounding nervous substance does not appear to be softened; the rest of the cerebellum and the spinal marrow are healthy. The optic and fifth pair of nerves do not present any change of structure.

Chest : Lining membrane of larynx and bronchi somewhat injected; some miliary tubercles in the lungs with points of lobular pneumonia on both sides of the chest; bronchial glands tuberculated; the abdomen does not contain a single tubercle.

\section{CASE XII.}

Imperfect hemiplegia ; gradual loss of sight; strabismus ; difficulty of articulating ; general dropsy ; death ; tubercles of the cerebellum, crus cerebelli, and pons Varolii.

John Morgue, 11 years of age, a boy of strong constitution, enjoyed excellent health until February, 1833, when, in consequence of fright, he was seized (according to his parents' account) with a brain fever, which lasted several weeks; during his convalescence the limbs became odematous, and the abdomen dropsical. In August last he came to Paris, and there the fluid was drawn off by puncture, which was again repeated in a month; after three weeks it was found necessary to puncture the abdomen once more, and in a few days the boy, who for some time had complained of a severe lancinating pain in the occiput, began to squint; his sight at the same time became feeble, and was occasionally lost; the mouth was drawn to one side; the articulation of sounds was impeded, and the sensibility of the left side much diminished. The strabismus and difficulty of speaking disappeared in six weeks but during that time the child had several attacks of vertigo and giddiness. The deviation of the moulh now became more marked every day; the sight more feeble; the right side almost completely paralysed, and infiltrated. Fluid again accumulated in the abdomen, and four quarts of serum were drawn off about the middle of Noveniber. In this state the child was admitted into hospital, when the following symptoms were noted:-

General paleners of the surface; pain in the back of the head; deviation of the mouth to the left side; incomplete paralysis of the same side, with diminurion of the sensibility; intellectual faculties unimpaired, but the articulation of sounds extremely difficult; sight much impaired; right pupil dilated; left one contracted; tongue moist; abdomen tumefied on each side; epigastrium and right lumbar region painful on pressure; seven or eight fluid stools in the twenty-four hours; they are fœtid, and passed involuntarily; skin dry and warm; pulse small, 124 ; considerable matity over the region of the heart, but no abnormal sound discoverable; cough frequent, without expectoration; respiration slightly impeded; mucous rále on both sides of the chest, with broncophony behind. During the next week the paralysis of the right side became gradually more complete, without any disturbance of the intellectual faculties; the state of the pupils was very variable, sometimes contracted. sometimes dilated; sometimes contraction of one with dilatation of the other, and vice versd. The headache was occasionally felt.

On the night of December 30th, the child was seized with violent delirium, with acute cries, soon followed by great prostration.

Jan. 1. Face pale and decomposed; pupils contracted; sight partially gone; pulse almost imperceptible; the tumefaction of the abdomen appears to have subsided. From the 1 st to the 5 th the little patient became every day more feeble, and died without convulsions on the night of the fifth.

\section{Body examined 30 hours after Death.}

Head: No fluid in the great cavity of the arachnoid, which appears to be free from change; but in the cellular tissue underneath, anil especially at the left side, there is an infiltration of gelatinous fluid, in some places clear, in others purple-red; the membranes do not adhere at any point, to the cerebral substance; a very small quantity of serum in the ventricles, the walls of which are not softened.

On the lateral and external parts of each lobe of the cerebellum there is a tubercle of the size of a bean; the surrounding nervous tissue is very slightly softened, but not injected; the middle lobe of the cerebellum also contains a small hard tubercle. 'The centre of the pons Varolii is occupied by a hard tubercle, as large as a walnut, which extends into the left crus cerebri; the right crus cerebelli contains another, not larger than a nut.

Chest : A small mass of tubercular matter at the summit of the left lung; the parenchyma of both studded with miliary tubercles; bronchial glands tubercular. The pericardium is considerably thickened, and false membranes, to the depth of two or three lines, cover its inner surface and the exterior of the heart, which latter organ, however, appears to be healthy.

Abdomen: The mucous membrane of the stomach is of a pale rose colour, and slightly softened near the bulging extremity; near the termination of the ileum, and in the valve of the colo:, are numerous ulcerations, which exist also, and in greater number, in the arch of the colon. Liver, pancreas, and spleen, healthy ; right kidney heulthy; the left kidney is transformed into a urinary cyst, and a calculus obstructs the water about three inclies from the pelvis, completely opposing the passage of the urine; hence the superior part of the ureter is as large as the small intestine; bladder healthy, except at the upper and posterior part, where there is sinall tubercular ulceration of the mucous membrane.

\section{CASE OF MONSTROSITY.}

Br S. N. PARSONS, Esq., Wincanton.

ON the 17 th of September, $1 \subseteq 38$, I was called on by a midwife to a poor woman in labour with her second child. On my arrival, I found that the fotus was expelled, and my attention was directed to an enormous cyst (adhering to the nates of the infunt), which had contained a considerable quantity of fluid. but was now empty and collapsed, the fluid liaving made its escape, partly during labour and the remainder subsequently to the birth of the infant, from a small aperture at the inferiur portion of the cyst, where its parieties were slightly sphacelated. From a suall quaritity which 1 had an opportunity of iuspecting. it appeared to be sero-sanguineous. The external covering of the cyst consisted of the common integument, but its parieties every where felt considerably thickened. There was but one funis and one placenta, about $w$ hich there was nothing abnormal. On visiting the infant 22 hours after birth. I found that it had passed meconium several times, but had not sucked or otherwise taken much nutrition. 'The spinal column was quite natural, and the inferior extremities could be moved with facility. The adventitious structure had now changed colour very considerably; in fact it was rapidly losing its vitality. At the end of 48 hours from the birth of the infant mortification was complete throughout the cyst, about which time the child likewise ceased to exist.

REMARKs.-Was this an abortire effort of nature to effect the formation of twins? That there was no congenital disease in the infant which bore any resemblance to the present case affords a negative proof in support of the supposition; and the abnormal structure having been covered by the common integument and continuous with the nates, affords, perhaps, an additional argument of its having been an embryo, but defective in its subsequent development. Müller, in his physiology, states that the formation of the double monster is caused by the force of organic attraction, and that similar parts have an affinity for each other: thus, in almost all cases on record, they are united by their corresponding parts; for example, face with face, either by the anterior or lateral surface; neck 